\title{
Phase-Contrast and Dark-Field Imaging
}

\section{Simon Zabler}

Fraunhofer Development Center X-ray Technology (EZRT) and Lehrstuhl für Röntgenmikroskopie (LRM), Josef-Martin Weg 63, 97084 Würzburg, Germany; simon.zabler@iis.fraunhofer.de

Received: 25 September 2018; Accepted: 25 September 2018; Published: 2 October 2018

\begin{abstract}
Very early, in 1896, Wilhelm Conrad Röntgen, the founding father of X-rays, attempted to measure diffraction and refraction by this new kind of radiation, in vain. Only 70 years later, these effects were measured by Ulrich Bonse and Michael Hart who used them to make full-field images of biological specimen, coining the term phase-contrast imaging. Yet, another 30 years passed until the Talbot effect was rediscovered for X-radiation, giving rise to a micrograting based interferometer, replacing the Bonse-Hart interferometer, which relied on a set of four Laue-crystals for beam splitting and interference. By merging the Lau-interferometer with this Talbot-interferometer, another ten years later, measuring $\mathrm{X}$-ray refraction and X-ray scattering full-field and in cm-sized objects (as Röntgen had attempted 110 years earlier) became feasible in every X-ray laboratory around the world. Today, now that another twelve years have passed and we are approaching the 125th jubilee of Röntgen's discovery, neither Laue-crystals nor microgratings are a necessity for sensing refraction and scattering by X-rays. Cardboard, steel wool, and sandpaper are sufficient for extracting these contrasts from transmission images, using the latest image reconstruction algorithms. This advancement and the ever rising number of applications for phase-contrast and dark-field imaging prove to what degree our understanding of imaging physics as well as signal processing have advanced since the advent of X-ray physics, in particular during the past two decades. The discovery of the electron, as well as the development of electron imaging technology, has accompanied X-ray physics closely along its path, both modalities exploring the applications of new dark-field contrast mechanisms these days. Materials science, life science, archeology, non-destructive testing, and medicine are the key faculties which have already integrated these new imaging devices, using their contrast mechanisms in full. This special issue "Phase-Contrast and Dark-field Imaging" gives us a broad yet very to-the-point glimpse of research and development which are currently taking place in this very active field. We find reviews, applications reports, and methodological papers of very high quality from various groups, most of which operate X-ray scanners which comprise these new imaging modalities.
\end{abstract}

Keywords: X-ray phase-contrast imaging; X-ray scattering; dark-field imaging; Computed Tomography; Talbot-interferometer; coded-aperture imaging; Moiré pattern analysis; Electron Backscatter imaging; cultural heritage; medical imaging; image processing; fourier image analysis

\section{An Introduction to This Special Issue}

Following the Bonse-Hart X-ray interferometer, laboratory grating-based X-ray phase-contrast and dark-field imaging were first demonstrated in 2006 by Franz Pfeiffer's group at Paul-Scherrer Institute (PSI), Switzerland [1,2]. In fact, the dark-field modality was discovered two years later by the same group [3]. These developments were merely a translation of the previously introduced "Talbot-interferometer", invented by Atsushi Momose and first demonstrated by Christian David in 2002 [4,5]. It is worth noting that both Momose and David did their early experiments at the European Synchrotron Radiation Facility (ESRF). It was on the ID-19 beamline of this facility where 
the principal development and proof-of-concept of differential phase contrast (DPC) imaging based on Talbot-interferometry took place from 2002 to 2006. Twelve years later, thanks to the addition of a third (G0) grating making the Talbot-interferometer a Talbot-Lau interferometer, which overcomes the restrictions on source coherence, more than 100 academic laboratories reportedly host Talbot-Lau interferometers, performing experiments of this kind on a daily basis. Phase-contrast and dark-field imaging are about to become standard techniques.

\subsection{Data, Methods and Results}

One of the groups which exploits these new modalities is the Biomaterials Science Center at the University of Basel, Switzerland, where Bert Müller et al. employ micro computed tomography (CT) as well as grating-based phase-contrast (GBPC) for studying carious lesions in teeth, more precisely in the enamel [6]. Enamel is a nanocrystalline and prismatic arrangement of hydroxyapatite crystallites. This material features a highly anisotropic, oriented microstructure. Through the combination of micro-CT with scanning small-angle X-ray scattering (SAXS), the authors show how the lesions correlate (or not) with the orientation of the underlying structure. In addition to the very visual insights into carious lesions, their paper paves the ground for future applications (clinical or preclinical) of X-ray vector radiography (XVR), which is based on the anisotropy in dark-field image contrast (DIC). DIC has been shown to encode the same signal as ultra-small angle X-ray scattering (USAXS), which originates from micro- and nanometer-sized scatterers.

Veronika Ludwig from Gisela Anton's group (ecap) at Erlangen Friedrich Alexander University (FAU), on the other hand, presents the potential application of DIC and DPC to examine archeological findings comprising fabric and organic remnants [7]. This study is very valuable, first because it establishes an absolute scale of DIC for a whole array of fabrics and woven structures; and second because it addresses the optimization of the measurement protocol for performing DIC and DPC for different specimens. The group has been working on dose sensitive clinical applications such as mammography for long. Since X-ray inspections of archeological findings, in particular organic remnants, have been declared a dose sensitive issue (the inspection must not destroy DNA remnants) as well, Veronika Ludwig's study makes a perfect starting point for this application.

On the methodological side, we received surprisingly many contributions which dealt with "alternative acquisition schemes" to measure DPC and DIC, and only few on the "classical acquisition scheme" for Talbot-Lau imaging. One reason for this may be that the classical acquisition scheme includes three line gratings, each of which has to be positioned and oriented with respect to the X-ray beam axis as well as with respect to each other, hence 9 translational and 9 rotational degrees of freedom, some of which can be omitted, but others not, making X-ray GBPC a relatively bulky and expensive experiment. GBPC is further limited in terms of X-ray energy by the available aspect rations of the intensity gratings, which already reach 1:100 by current state-of-the-art LIGA (lithography and galvanic molding). An outstanding review on X-ray speckle-based imaging, written by Marie-Christine Zdora, a former student of Irene Zanette, from Diamond Light Source, is setting the ground for obtaining phase-contrast and dark-field data without using line gratings [8]. Speckle-based imaging is presented as a "single-shot" (XST) as well as a "stepping" (XSS) technique. These methods replace the grating with a simple diffusor, which may be sandpaper or a fibrous membrane. Unlike the conventional Talbot-Lau setup, XST and XSS yield two-dimensional DPC and DIC contrast.

The same methodological bifurcation, from stepping to single-shot, is reported by Maria Seifert from FAU. Seifert and her coauthors set the stage (meaning, the line gratings) to obtain very regular fine-spaced moiré fringes. The latter can also be analyzed from a single-shot, yielding DPC and DIC images at the expense of spatial resolution. While for XST this spatial resolution is linked to the speckle size, which has to be carefully adjusted, the improved image reconstruction by Seifert et al., from moiré patterns yields a relatively homogeneous resolving power. Distortions of the patterns caused by bent gratings are compensated for by their improved data processing chain [9]. 
Extending spackle-based imaging, in particular X-ray speckle tracking (XST), to higher energies in laboratory settings is the ambitious goal of Tunhe Zhou and Fei Yang, a close collaboration between the Swiss EMPA (Eidgenössische Materialprüfungs- und Forschungsanstalt) and Diamond Light Source, UK [10]. Replacing the "standard" sandpaper which has been often used as diffusor for XST with steel wool, Zhou and her coauthors produce a random polychromatic speckle pattern in cone beam geometry with an average visibility of $17 \%$ at $80 \mathrm{kVp}$ acceleration voltage, which is outstandingly good. The study shows applications of the technique to electronics (2D inspection) and to mortar (3D CT). These results are very impressive and extending their setup to XSS in addition to XST would indeed greatly improve spatial resolution.

Another nonconventional technique, namely Edge Illumination (EI), has evolved simultaneously to the Talbot-Lau interferometer, yielding images very similar to DIC and DPC. The report from Marco Endrizzi from Alessandro Olivio's group at University College London (UCL) is the latest hallmark in data analysis from EI imaging [11]. Endrizzi's method first reduces pixel sampling by binning to avoid artifacts which arise from high-frequency noise. Then, higher modulation amplitude and phase are progressively reconstructed by reducing the binning size until the desired pixel resolution is achieved. The result is a very stable reconstruction algorithm which also applies to single-shot EI image acquisition (here line scanning was used) and possibly to Talbot-Lau interferometers as well.

Jonas Dittmann from LRM (Lehrstuhl für Röntgenmikroskopie) Würzburg, Germany, presents a new algorithm which resolves a similar issue in Talbot-interferometry: The instrument, having very strong angular sensitivity with respect to the gratings positions, is commonly suffering from mechanical and thermal instabilities, either as a result of thermal drift or wobble of the phase-stepping axis [12]. Therefore, for example, during a CT scan, reference images are taken repeatedly and often. Dittmann uses the moiré patterns themselves to introduce a numerical self-alignment of the gratings positions and orientations. Thus, common artifacts, for example, residual moiré patterns, are eliminated and quality is improved. The algorithm is also well suited for a fine adjustment of the interferometer itself.

Lastly, Nicolas Brodusch from McGill University, Montréal Canada, guides us to a different use of the term dark-field imaging, thus widening the scope of this special issue beyond the domain of X-ray physics, namely to electron imaging. Brodusch's review on Electron Backscatter Diffraction (EBSD) and EBSD-DF (dark-field) imaging includes a very appealing explanation of how the BS electron diffraction patterns (EBSP) originate and are processed to yield a two-dimensional map of the lattice structure in polycrystalline materials. Just like the X-ray DIC, the darkfield contrast was only recently discovered in EBDS by selecting a small (dark) region in pseudo-Kikuchi diffraction patterns and plotting average intensity as a function of the electron spot coordinates. Today, EBSD-DF is gradually explored and recognized to encode very valuable physical information, for example, materials composition and/or surface topography (depending on the collection angle of the virtual aperture). Brodusch and his coauthors apply EBSD-DF to study minerals as well as magnetic domains in electrical steel, both in reflection. When EBSD-DF is applied in transmission (AA2099 Al-Li-Cu alloy), the visual effects which arising from this contrast mode are even more pronounced, revealing additional information about precipitates and deformation in the alloy's grain structure [13].

\subsection{Quality and Impact}

The eight research papers which were published in this special issue between March 2018 and August 2018, all with very short time spans between submission, reviews, and acceptance, feature a very high level of research and reporting quality. The manuscripts were reviewed quickly yet extensively by known experts in the field of X-ray imaging and electron imaging all throughout the world.

\section{Further Reading}

Readers interested in the topic of phase-contrast and darkfield imaging are referred to the book of David Paganin "Coherent X-ray optics" as well as to the pioneering publications by Atsushi Momose, 
Christian David, and Franz Pfeiffer [1-4,14]. For EBSD imaging, one might refer to the book by Schwartz et al. [15].

Funding: This research received no external funding.

Acknowledgments: The guest editor thanks all contributing authors for dedicating their work and time to this special issue as well as the anonymous peer reviewers for their prompt yet elaborate remarks, who were keeping the presentation standard for this issue constantly high. Assistant Editor Veronica Wang, a lot of thanks for her tireless efforts and communications throughout the editing process.

Conflicts of Interest: The author declares no conflict of interest.

\section{References}

1. Bonse, U.; Hart, M. An X-ray interferometer. Appl. Phys. Lett. 1965, 6, 155-156. [CrossRef]

2. Pfeiffer, F.; Weitkamp, T.; Bunk, O.; David, C. Phase retrieval and differential phase-contrast imaging with low-brilliance X-ray sources. Nat. Phys. 2006, 2, 258-261. [CrossRef]

3. Pfeiffer, F.; Bech, M.; Bunk, O.; Kraft, P.; Eikenberry, E.F.; Brönnimann, C.; Grünzweig, C.; David, C. Hard-X-ray dark-field imaging using a grating interferometer. Nat. Mater. 2008, 7, 134-137. [CrossRef] [PubMed]

4. Momose, A.; Kawamoto, S.; Koyama, I.; Hamaishi, Y.; Takai, K.; Suzuki, Y. Demonstration of X-ray Talbot interferometry. Jpn. J. Appl. Phys. 2003, 42, L866-L868. [CrossRef]

5. David, C.; Nöhammer, B.; Solak, H.; Ziegler, E. Differential X-ray phase contrast imaging using a shearing interferometer. Appl. Phys. Lett. 2002, 81, 3287-3289. [CrossRef]

6. Deyhle, H.; White, S.; Botta, L.; Liebi, M.; Guizar-Sicairos, M.; Bunk, O.; Müller, B. Automated Analysis of Spatially Resolved X-ray Scattering and Micro Computed Tomography of Artificial and Natural Enamel Carious Lesions. J. Imaging 2018, 4, 81. [CrossRef]

7. Ludwig, V.; Seifert, M.; Niepold, T.; Pelzer, G.; Rieger, J.; Ziegler, J.; Michel, T.; Anton, G. Non-Destructive Testing of Archaeological Findings by Grating-Based X-Ray Phase-Contrast and Dark-Field Imaging. J. Imaging 2018, 4, 58. [CrossRef]

8. Zdora, M.C. State of the Art of X-ray Speckle-Based Phase-Contrast and Dark-Field Imaging. J. Imaging 2018, 4, 60. [CrossRef]

9. Seifert, M.; Gallersdörfer, M.; Ludwig, V.; Schuster, M.; Horn, F.; Pelzer, G.; Michel, T.; Anton, G. Improved Reconstruction Technique for Moiré Imaging Using an X-Ray Phase-Contrast Talbot-Lau Interferometer. J. Imaging 2018, 4, 62. [CrossRef]

10. Zhou, T.; Yang, F.; Kaufmann, R.; Wang, H. Applications of Laboratory-Based Phase-Contrast Imaging Using Speckle Tracking Technique towards High Energy X-Rays. J. Imaging 2018, 4, 69. [CrossRef]

11. Endrizzi, M.; Vittoria, F.; Olivo, A. Single-Shot X-ray Phase Retrieval through Hierarchical Data Analysis and a Multi-Aperture Analyser. J. Imaging 2018, 4, 76. [CrossRef]

12. Dittmann, J.; Balles, A.; Zabler, S. Optimization based evaluation of grating interferometric phase stepping series and analysis of mechanical setup instabilities. J. Imaging 2018, 4, 77. [CrossRef]

13. Brodusch, N.; Demers, H.; Gauvin, R. Imaging with a Commercial Electron Backscatter Diffraction (EBSD) Camera in a Scanning Electron Microscope: A Review. J. Imaging 2018, 4, 88. [CrossRef]

14. Paganin, D. Coherent X-ray Optics; Oxford Science Publications: New York, NY, USA, 2006.

15. Schwartz, A.J.; Kumar, M.; Adams, B.L.; Field, D.P. Electron Backscatter Diffraction in Materials Science; Springer: New York, NY, USA, 2009.

(C) 2018 by the author. Licensee MDPI, Basel, Switzerland. This article is an open access article distributed under the terms and conditions of the Creative Commons Attribution (CC BY) license (http:// creativecommons.org/licenses/by/4.0/). 